\title{
COVID-19 cohort on children with cancer: delay in treatment and increased frequency of deaths
}

Ana Luiza Magalhães de Andrade Lima 1

https://orcid.org/0000-0003-0016-2854

Maria do Céu Diniz Borborema 2

iD https://orcid.org/0000-0003-4245-3350

Ana Paula Rodrigues Matos 3

https://orcid.org/0000-0003-3908-4304

Kaline Maria Maciel de Oliveira 4

iD https://orcid.org/0000-0002-7549-1495
Maria Júlia Gonçalves Mello 5

(iD) https://orcid.org/0000-0003-4645-8343

Mecneide Mendes Lins 6

iD https://orcid.org/0000-0002-9155-5030

1-6 Instituto de Medicina Integral Prof. Fernando Figueira. Rua dos Coelhos, 300. Boa Vista. Recife, PE, Brasil. CEP: 50.070-902. E-mail: analuizadealima@gmail.com

\begin{abstract}
Objectives: to describe epidemiological characteristics and deaths in children with cancer and COVID-19 at a reference hospital in Recife, Brazil.

Methods: cohort involving children under the age of 19 underwent cancer treatment during April to July 2020. During the pandemic, real-time reverse transcriptase polymerase chain reaction assay (RT-PCR) for severe acute respiratory syndrome coronavirus 2 (SARS CoV-2) in nasal / oropharyngeal swab were collected in symptomatic patients or before hospitalization. Those with detectable results were included in this cohort study. The outcomes were delayed on cancer treatment and death. Descriptive analysis was performed and presented in preliminary results.

Results: 48 children participated in the cohort, mostly with hematological neoplasms (66.6\%.),69\% were male, median age was 5.5 years. The most frequent symptoms were fever (58.3\%) and coughing (27.7\%); $72.9 \%$ required hospitalization, $20 \%$ had support in ICU and $10.5 \%$ on invasive ventilatory assistance. $66.6 \%$ of the patients had their oncological treatment postponed, $16.6 \%$ died within 60 days after confirmation of SARS-CoV-2 infection.

Conclusions: COVID-19 led a delay in the oncological treatment for children with cancer and a higher mortality frequency when compared to the historical series of the service. It would be important to analyze the risk factors to determine the survival impact.
\end{abstract}

Key words COVID-19, Child, Mortality, Neoplasms, Coronavirus infections 


\section{Introduction}

Adult cancer patients infected with the severe acute respiratory virus coronavirus 2 (SARS-CoV-2) tend to have more severe clinical outcomes when compared to non-oncologic patients, highlighting the hematological, lung and metastatic cancers stages. ${ }^{1}$ Despite the lack of data, recent articles suggest that the morbidity of the new coronavirus 2019 disease (COVID-19) in pediatric patients with cancer is low, and that these patients are no longer vulnerable to SARS-CoV-2 infection when compared to children without comorbidities. 2,3

In general, children get less sick, that is, they have less susceptibility and when they become infected, there is a predominance of asymptomaticor mild cases, so it is less severe.4-6 The most common symptoms in the acute phase are coughing and fever, and a minority needs hospitalization and support in the intensive care unit (ICU), with a complication rate of less than $2 \%$. However, cases of greater severity called multi-systemic inflammatory syndrome associated with COVID-19 (SIM-C) have been described and are considered a late complication in the pediatric age group. 7

Most pediatric cancers behave aggressively, requiring immediate treatment and may need long periods of intensive chemotherapy with multiple antineoplastic agents. 8 In contrast, they respond better to the treatment and are considered of good prognosis when compared to adults. Therefore, in addition to the concern with SARS-CoV-2 infection in children with cancer, there is a fear of delay in the diagnosis and treatment of these patients due to the difficulties caused by the pandemic. 9

In this context, it is important to establish a cohort of pediatric cancer patients in various stages of the treatment in order to determine the burden of the disease in this population and its clinical evolution. 10,11 Preliminary data from a cohort evaluating the repercussions on treatment delay and the frequency of deaths of any cause are presented in this article.

\section{Methods}

Cohort type study, involving pediatric patients in cancer treatment at the Instituto de Medicina Integral Prof. Fernando Figueira (IMIP), in Recife, PE, during the period of April 1 to July 31, 2020, at a time of great circulation of SARS-CoV-2 virus in the State of Pernambuco, Brazil.

IMIP is a quaternary hospital, a reference on oncological treatment for patients from the Public
Health System (Sistema Unico de Saúde-SUS). The Pediatric Oncology Service, opened since 1994, has an average of 250 patients/month in chemotherapy treatment and assists about 60 patients/day in the outpatient consultation. Depending on the oncological treatment phase, these children return to the service for consultation and/or procedures two or three times a week and even daily. Patients who do not live in the metropolitan region in Recife, stay in a 'support home' during the oncological treatment.

Due to the pandemic, a protocol to collect nasal/oropharyngeal swab for SARS-CoV-2 research through polymerase chain reaction in real-time reverse transcription (RT-PCR) has been established for all symptomatic patients or during hospital admission and/or invasive procedures.

Patients under 19 years old or up to three months at the end of oncological treatment with detectable results for SARS-CoV-2 performed by RT-PCR were included.

The data were collected by the pediatric oncology team, on a specific form, through information from the medical records and interviews were performed by a person in charge. Sociodemographic variables (age, sex, weight at birth, comorbidities), clinical aspects of the baseline (signs and symptoms that could be associated with COVID-19, type of cancer and oncological treatment phase, severity classification) and evolution were evaluated. The outcomes studied were delay on the oncological treatment and death.

The capture was done consecutively in the assistance sector or hospitalized. A database was built in the Excel program, cleaning and correction of eventual inconsistencies were performed. The analysis of the descriptive data was carried out on Stata13.1, (StataCorp, College Station, United States) establishing measures of central tendency and frequency distribution according to the characteristics of the variables referring to the sample described.

This research is part of a larger project entitled "Cohort on COVID-19 in newborns, children and adolescents: epidemiological characteristics, clinical laboratory, genome of SARS COV-2 and the dynamics of viral load and immunological markers associated with prognosis" approved by CEP at IMIP, CAAE number: 31578520.8.0000.5201. The authors declared no conflicts of interest.

\section{Results}

During the study period, 151 RT-PCR exams were performed for SARS-CoV-2 research in 137 patients with pediatric cancer; of these 54 had detectable 
results and six did not fulfill the inclusion criteria, thus 48 patients were enrolled in the cohort.

Most of the participants (69\%) were male with a median age of 5.5 years old (Table 1). Cancer types, $66.6 \%$ were hematological neoplasms with a predominance of leukemia $(64.5 \%)$ and one patient (2\%) had Burkitt's lymphoma. Of the solid tumors, the neuroblastoma was the most prevalent, present in four $(8.4 \%)$ patients.

No comorbidities were evidenced such as neurological diseases or diabetes mellitus, but two patients had asthma (4\%), one had Fanconi syndrome and six $(12.5 \%)$ were obese (BMI score $\mathrm{z}>3.0$ for age). A history of low birth weight $(<2.5 \mathrm{~kg})$ was reported on two patients $(4 \%)$ of the sample, although this data was not present in five forms.

Contact with COVID-19 at home or at a support home where patients were staying was reported in eight $(16.6 \%)$ of the cases. Of the 48 patients, eight $(16.6 \%)$ were asymptomatic. However, most of the sample $(68 \%)$ presented up to three symptoms, mainly fever (58.3\%), coughing $(27.7 \%)$ and runny nose $(23.4 \%)$. Eight participants $(16.6 \%)$ presented new episodes of fever after more than 24 hours.

Approximately $3 / 4$ of the patients $(72.9 \%)$ required hospitalization, $10(20.1 \%)$ required ICU admission and oxygen supplementation, and five $(10 \%)$ needed invasive respiratory support associated with hemodynamic support with vasoactive drugs.

More than half $(66.6 \%)$ of the patients had a delay in the oncological treatment cycle in the presence of SARS-CoV-2 infection, with a median of 15 days in leukemia and 22.5 days in solid tumors. (Table 2) The only patient with Burkitt's lymphoma had no delay in the oncological treatment.

Five $(10.5 \%)$ participants of this study died within 30 days after RT-PCR SARS-CoV-2 collection, while eight $(16.6 \%)$ died within 60 days after diagnosis of COVID-19. Six (12.5\%) patients that died were in the induction phase of the oncological treatment and seven $(14.6 \%)$ were male.

\section{Discussion}

The present study showed a delay in the oncological treatment, besides a greater need for admission in the pediatric ICU and deaths, when compared to the current evidence of children with COVID-19 with ${ }^{2,3,9,12}$ or without cancer.4-6 As we presented only preliminary descriptive data, it was not possible to analyze the risk factor.

Nasal/oropharyngeal swab collection for RTPCR SARS-CoV-2 was performed in more than half of the pediatric population under the oncological treatment of the service and infection detected in $1 / 3$ of the patients. This data reinforces the value of this study and the sample size was larger than the case series carried out in Italy and Spain (Madrid) and the American cohort (New York); the largest of these studies included 29 pediatric patients with cancer and COVID-19.2,3,9,12

The types of cancers found match the reports in the literature regarding pediatric oncology, where leukemia predominates $2,3,9,12$ and differs from that observed in adults, with greater frequency of solid tumors. ${ }^{1,11,13}$ This can be justified, since leukemia is the most prevalent neoplasm in the pediatric age group. 14

There was a predominance of male individuals, also observed in other studies involving oncologic 1,11 adult and pediatric patients with cancer.2,12 Although some studies show a higher incidence of COVID-19 in females, ${ }^{3,9}$ more severe forms of the disease have been documented in male children and adolescents. 10

Despite the literature report that COVID-19 presents asymptomatically or with mild symptoms in most children, $20 \%$ required hospitalization 4,5 and low mortality, 4,15 including those with cancer;2,3,9,12 the present study showed a predominance of patients with symptoms and the need for hospitalization, ICU and ventilatory support. An interpretation of the results would be the most severe clinical profile of the patients in the sample, all in the oncological treatment, most in the induction phase, characterizing a greater need to come and go in the hospital environment. It differs from the series of cases by Madrid,2 in which cancer patients were included, regardless of the treatment. Another interpretation would be the performance of RT-PCR in patients with COVID-19 symptoms or who would need hospitalization for other reasons.

It is important to emphasize that, in the last five years, early mortality - in the oncological treatment induction phase - was less than $2 \%$ in this pediatric oncology service. However, in the current year, SARS-CoV-2 infection has increased the frequency of deaths by more than ten times.

In addition, most patients had their cancer treatment postponed, which, added to the complications of COVID-19 and hospital admission, may have contributed to the higher frequency of deaths in the sample. We emphasize that the interruption on the oncological treatment is a cause of great concern for pediatric oncologists and may contribute negatively for the chances of curing the patients.

This publication is the descriptive part of the 
Table 1

Distribution of biological characteristics, type of tumor and treatment phase, clinical symptoms possibly associated with COVID-19 and clinical evolution of the cohort on 48 pediatric patients with cancer and detectable RT-PCR SARSCoV-2 followed at IMIP Pediatric Oncology, April to July 2020.

\begin{tabular}{|c|c|c|}
\hline Variables & $\mathbf{N}$ & $\%$ \\
\hline \multicolumn{3}{|c|}{ Biological variables and related to neoplasm } \\
\hline \multicolumn{3}{|l|}{ Age (years) } \\
\hline Extremes & \multicolumn{2}{|c|}{$0.6-18.6$} \\
\hline $\bar{X} \pm S D$ & \multicolumn{2}{|c|}{$6.2 \pm 4.5$} \\
\hline Median (interquartile range - IIQ) & \multicolumn{2}{|c|}{$5.5(3-8)$} \\
\hline \multicolumn{3}{|l|}{ Sex } \\
\hline Male & 33 & 69.0 \\
\hline Female & 15 & 31.0 \\
\hline \multicolumn{3}{|l|}{ Neoplasm } \\
\hline Leukemia & 31 & 64.5 \\
\hline Solid Tumores & 16 & 33.5 \\
\hline Lymphoma & 1 & 2.0 \\
\hline \multicolumn{3}{|l|}{ Treatment phase } \\
\hline Induction & 23 & 48.0 \\
\hline Maintenance / reinduction & 18 & 37.6 \\
\hline Consolidation & 4 & 8.3 \\
\hline Palliative care & 2 & 4.1 \\
\hline Post surgery & 1 & 2.0 \\
\hline \multicolumn{3}{|l|}{ Clinical variables and evolution } \\
\hline \multicolumn{3}{|l|}{ Symptoms /signs } \\
\hline Asymtomatic & 8 & 16.6 \\
\hline Fever & 28 & 58.3 \\
\hline Mialgia & 2 & $4 . .3$ \\
\hline Cephalea & 4 & 8.5 \\
\hline Runny nose & 11 & 23.4 \\
\hline Odinophagia & 3 & 6.4 \\
\hline Coughing & 13 & 27.7 \\
\hline Respiratory discomfort & 10 & 20.8 \\
\hline Diarrheia & 10 & 20.8 \\
\hline Nausea, vomiting & 2 & 4.3 \\
\hline Abdominal pain & 2 & 4.3 \\
\hline Anosmia & 4 & 8.5 \\
\hline Ageusia & 1 & 2.1 \\
\hline Oral mucosal injury & 3 & 6.4 \\
\hline Skin injury & 3 & 6.4 \\
\hline Petechial & 2 & 4.3 \\
\hline Equimose & 2 & 4.3 \\
\hline \multicolumn{3}{|l|}{ Service during follow-up } \\
\hline Hospitalization & 35 & 72.9 \\
\hline Outpatient & 13 & 27.1 \\
\hline \multicolumn{3}{|l|}{ Ventilatory support } \\
\hline No & 38 & 80.9 \\
\hline Yes & 10 & 19.1 \\
\hline * Non-invasive & 5 & 10.2 \\
\hline *Invasive & 5 & 10.2 \\
\hline
\end{tabular}

IMIP= Instituto de Medicina Integral Prof. Fernando Figueira. 
Table 2

Delay in the treatment and deathsof any cause in this cohort on 48 pediatric cancer patients and RT-PCR SARS-CoV-2 detectable accompanied at the Pediatric Oncology Service of IMIP from April to July 2020.

\begin{tabular}{|c|c|c|}
\hline Delay in the treatment / deaths & $\mathbf{N}$ & $\%$ \\
\hline \multicolumn{3}{|l|}{ Delay in the treatment } \\
\hline Yes & 32 & 66.6 \\
\hline No & 16 & 33.4 \\
\hline \multicolumn{3}{|l|}{ Patients with Leukemia (days of delay) } \\
\hline $\bar{X} \pm S D$ & \multicolumn{2}{|c|}{$15 \pm 8.8$} \\
\hline Median (interquartile range - IIQ) & \multicolumn{2}{|c|}{$15(9-21)$} \\
\hline \multicolumn{3}{|c|}{ Patients with solid tumors(days of delay) } \\
\hline $\bar{X} \pm S D$ & \multicolumn{2}{|c|}{$18.3 \pm 20$} \\
\hline Median (interquartile range - IIQ) & \multicolumn{2}{|c|}{$22.5(14-44)$} \\
\hline \multicolumn{3}{|l|}{ Deaths } \\
\hline$<30$ days & 5 & 10.4 \\
\hline 30 a 60 days & 3 & 6.4 \\
\hline \multicolumn{3}{|l|}{ Death on patients with leukemia } \\
\hline Yes & 4 & 12.9 \\
\hline No & 27 & 87.1 \\
\hline \multicolumn{3}{|l|}{ Deaths on patients with solid tumors } \\
\hline Yes & 4 & 25.0 \\
\hline No & 12 & 75.0 \\
\hline
\end{tabular}

IMIP= Instituto de Medicina Integral Prof. Fernando Figueira.

cohort, which reinforces the importance of the analysis on the risk factors associated with these outcomes and the analysis of children's survival on oncological treatment with COVID-19.

\section{Authors' contribution}

Lima ALMA, Mello MJG, Lins MM contributed with the design, planning, table, data analysis and interpretation, writing and reviewing articles. Oliveira KMM contributed with the conception, planning, data analysis and interpretation, writing and reviewing the article. Borborema, MCD, Matos, ANR contributed with data collection, writing and reviewing of the article. All authors approved the final version of the article. 


\section{References}

1. Dai M, Liu D, Liu M, Zhou F, Li G, Chen Z, et al. Patients with cancer appear more vulnerable to SARS-CoV-2: A multicenter study during the COVID-19 outbreak. Cancer Discov. 2020; 10 (6): 783.

2. Rojas T, Pérez-Martínez A, Cela E, Baragaño M, Galán V, Mata C, et al. COVID-19 infection in children and adolescents with cancer in Madrid. Pediatr Blood Cancer. 2020; 67 (7): 19-21.

3. Terenziani M, Massimino M, Biassoni V, Casanova M, Chiaravalli S, Ferrari A, et al. SARS-CoV-2 disease and children under treatment for cancer. Pediatr Blood Cancer. 2020; 67 (9): 1-2.

4. Yoon S, Li H, Lee KH, Hong SH, Kim D, Im H, et al. Clinical characteristics of asymptomatic and symptomatic pediatric coronavirus disease 2019 (Covid-19): A systematic review. Med. 2020; 56 (9): 1-14.

5. Lu X, Zhang L, Du H, Zhang J, Li YY, Qu J, et al. SARSCoV-2 infection in children. N Engl J Med. 2020; 382 (17): 1663-5.

6. Dong $\mathrm{Y}, \mathrm{Mo} \mathrm{X}, \mathrm{Hu} \mathrm{Y}$, Qi X, Jiang $\mathrm{F}$, Jiang $\mathrm{Z}$, et al Epidemiology of COVID-19 among children in China. Pediatrics. 2020; 145 (6): e20200702.

7. Xu S, Chen M, Weng J. COVID-19 and Kawasaki disease in children. Pharmacol Res. 2020; 159: 104951.

8. Kotecha RS. Challenges posed by COVID-19 to children with cancer. Lancet Oncol. 2020; 21(5): e235.

9. Bisogno G, Provenzi M, Zama D, Tondo A, Meazza C, Colombini A, et al. Clinical Characteristics and Outcome of Severe Acute Respiratory Syndrome Coronavirus 2 Infection in Italian Pediatric Oncology Patients: A Study From the Infectious Diseases Working Group of the Associazione Italiana di Oncologia e Ematologia Pediatrica. J Pediatric Infect Dis Soc [Internet]. 2020 Jul 11; Available from https://academic.oup.com/jpids/advancearticle/doi/10.1093/jpids/piaa088/5870367
10. Chao JY, Derespina KR, Herold BC, Goldman DL, Aldrich $\mathrm{M}$, Weingarten $\mathrm{J}$, et al. Clinical Characteristics and Outcomes of Hospitalized and Critically Ill Children and Adolescents with Coronavirus Disease 2019 at a Tertiary Care Medical Center in New York City. J Pediatr [Internet]. 2020;223:14-19.e2

11. Weinkove R, McQuilten ZK, Adler J, Agar MR, Blyth E, Cheng AC, et al. Managing haematology and oncology patients during the COVID-19 pandemic: interim consensus guidance. Med J Aust. 2020; 212 (10): 481-9.

12. Ferrari A, Zecca M, Rizzari C, Porta F, Provenzi M, Marinoni M, et al. Children with cancer in the time of COVID-19: An 8-week report from the six pediatric oncohematology centers in Lombardia, Italy. Pediatr Blood Cancer. 2020; 67 (8): 3-5.

13. ElGohary GM, Hashmi S, Styczynski J, Kharfan-Dabaja MA, Alblooshi RM, de la Cámara R, et al. The risk and prognosis of COVID-19 infection in cancer patients: A systematic review and meta-analysis. Hematol Oncol Stem Cell Ther. 2020; S1658-3876(20)30122-9.

14. Lins MM, Santos MO, Albuquerque MFPM, Castro CCL, Mello MJG, de Camargo B. Incidence and survival of childhood leukemia in Recife, Brazil: A population-based analysis. Pediatr Blood Cancer. 2017; 64 (8): e26391.

15. Patel NA. Pediatric COVID-19: Systematic review of the literature. Am J Otolaryngol - Head Neck Med Surg. 2020; 41 (5): 102573.

Received on October 1, 2020

Approved on November 26, 2020 